\title{
OPTIMIZING DETECTION OF ROAD FURNITURE (POLE-LIKE OBJECTS) IN MOBILE LASER SCANNER DATA
}

\author{
D. $\mathrm{Li} *$, S. Oude Elberink \\ Faculty of Geo-Information Science and Earth Observation, University of Twente, Netherlands - (li29820, \\ oudeelberink@itc.nl) \\ Commission III, WG III/2
}

KEY WORDS: Laser Scanning, LIDAR, Point Cloud, Segmentation, Classification, Processing, Algorithm

\begin{abstract}
:
Due to the road safety problem is becoming more and more serious recent years, existing road safety assessment by using automatic method is necessary. Meanwhile, since the pole-like objects have large effect on road safety and are in high demand as facilities to be managed, the automatic pole-like objects extraction is becoming a hot issue. As a result, a robust, quick and automatic pole-like object detection algorithm in MLS data is proposed in this paper. Two datasets are tested to show performance of the proposed algorithm, it demonstrates that it is feasible to detect tree with an overall accuracy of over $92 \%$ and other pole-like object of $72 \%$ in dataset $\mathrm{A}$ and $82 \%$ of tree points and $75 \%$ of other pole points in dataset $\mathrm{B}$.
\end{abstract}

\section{INTRODUCTION}

The road safety is influenced by three main factors such as: vehicle, driver behaviour and road environment or infrastructure (Mc Elhinney et al., 2010). In which, road infrastructure safety assessment is crucial to improve the transportation safety. In order to implement the road safety inspection, 3D feature modelling and extraction from laser data or imagery is adopted. Since the pole-like objects stand out from the road environment and have large effect on road safety problem, they play an important role in road environment. As a result, extracting polelike objects on the road side is necessary. The features are common in road environment and include different kinds of pole, e.g., traffic sign, lamp post. Moreover, the tree trunk can be also considered as pole-like objects.

Many current algorithms on pole-like object detection in mobile laser scanner data have been proposed. However, a robust, quick and fully automatic technique is still lacking. In this paper, the main research is based on an existing method proposed by (Pu et al., 2011). Due to some types of pole-like objects have similar appearance in point cloud and part of tree trunks are occluded by leaves which lead to no pole-like parts are visible, it is hard to get high detection accuracy and furthermore distinguish different types of pole accurately with current algorithm. Furthermore, only the coordinate information of laser point was used now. However in fact, most of the traffic signs are painted with special material of high reflectivity for better visualization, thus the reflectance strength of laser scanner data would be beneficial to algorithm improvement. In addition to this, since the significant difference between natural and manmade objects surface characteristics, to our expectation, the pulse count and segment number value can provide much richer information to differentiate between these two kinds of objects.

This research is expected to deliver a classified point cloud, in which each object is represented as one component. So each laser point will get a class label and a component number. The expectation is that all of pole-like objects can be detected correctly, and no objects are false detected. Then all of the detected pole-like objects can be classified into subclasses that specify the different types of pole.

This paper is divided into 5 sections. Section 2 reviews the previous pole-like objects extraction methods. Section 3 describes the proposed methodology based on the existing algorithm. Section 4 describes and discusses the result of proposed algorithm. Final conclusion and recommendation for further study are presented in section 5 .

\section{RELATED WORK}

Airborne, terrestrial and mobile laser scanners obtain large amount of laser points for the object extraction. This increases the potential finding objects in the urban environment, as can be seen by the list of researches.

\subsection{Segmentation}

In order to avoid extra computational consuming, segmentation is often the first step with regard to extract objects in laser point cloud (Vosselman et al., 2010), in which, surface growing segmentation is utilized to group nearby points based on the homogeneity criterion such as planar or smooth surface. Seed detection and surface growing are the two steps. Vosselman et al. (2004) review different kinds of segmentation techniques such as surface growing algorithm, scan line segmentation and connected component analysis as well as recognition of specific geometric shapes. (Rabbani et al., 2006) compare several segmentation methods and indicate the limitations of each segmentation method, then a segmentation method use smoothness constraint is presented.

\subsection{Tree detection}

Since tree trunks are also recognized as pole-like objects in this paper and tree detection accuracy is very low with current algorithm, several tree detection methods are reviewed. In (Darmawati, 2008), due to the characters of objects surface, the pulse count information is used to separate trees from other man-made objects. (Rutzinger et al., 2010) present a workflow

\footnotetext{
* Corresponding author
} 
for detecting and modelling trees in mobile laser point clouds, which consists of tree detection, tree simplification and tree modelling. (Yu et al., 2011) present an approach for predicting individual tree attributes, by using the physical and statistical features such as tree height, the diameter on breast height (DBH) in airborne laser scanner data.

\subsection{Pole-like objects detection}

(Brenner, 2009) present an intuitive approach to indicate linear structure if eigenvalues analysis yields one large and two small eigenvalues. But it would cause false detection when people standing close to the pole. Golovinskiy et al. (2009) propose a four steps method to recognizing objects of urban environments: locating, segmentation, characterizing and classify clusters of 3D points. A typical scan line segmentation is utilized in Lehtomäki et al. (2010) to remove long groups and keep short segments that are possible sweeps of pole. Then the clusters that belong to same poles are merged and classified as pole or nonpole through comparing with detection constrains. K-nearest neighbours graph, endpoint preserving Laplacian smoothing and Principle Component Analysis (PCA) are performed in Yokoyama et al. (2011) to recognize pole-like objects on correctly created segments. In the paper of El-Halawany et al. (2011), a pipeline for point cloud process to detect poles and find their dimensions from unorganized point clouds captured from a kinematic terrestrial laser scanner is proposed. In (Vakautawale, 2010), a detection algorithm based on 2D enclosing circle algorithm is proposed, which consists of four phases: segmentation, multiple filtering criteria, 2D enclosing circle algorithm and supervised classification.

The existing algorithm which will be analysed and improved in this research is proposed by ( $\mathrm{Pu}$ et al., 2011). Three phases are included in this algorithm: rough classification, percentile-based detection and further classification. Firstly, segmentation and filtering criteria are applied to remove the unwanted segments (e.g., building, car, bus platform). Secondly, percentile-based algorithm checks feature values based on one section from the component to determine whether the component is pole-like object. At last, knowledge-based shape recognition further classifies the already detected pole-like objects. Quantitatively evaluation of the proposed algorithm indicated that $20 \%$ of bare poles, $63.5 \%$ of trees, $60.8 \%$ of traffic sign and $81.8 \%$ of other poles were detected. Due to the low detection accuracy, we expect that this proposed pole detection approach can enhance at several critical steps by integrating an improved tree detection and automated quality evaluation, which will be described and analysed in detail in section 3 .

\section{METHODOLOGY}

Based on the existing algorithm, the methodology used in this research is proposed with respect to five phases: rough classification, rule-based tree detection, percentile-based pole extraction, and knowledge-based further classification; at last, quantitative assessment works cyclically to check the performance of proposed algorithm, which includes two parts: an automatic quality assessment based on point wise and a visual examination based on object wise. If the classified result is not satisfied enough, the optimization would start again from the beginning and continue to loop till getting the optimal result. Figure 1 shows the workflow of five-step proposed algorithm.

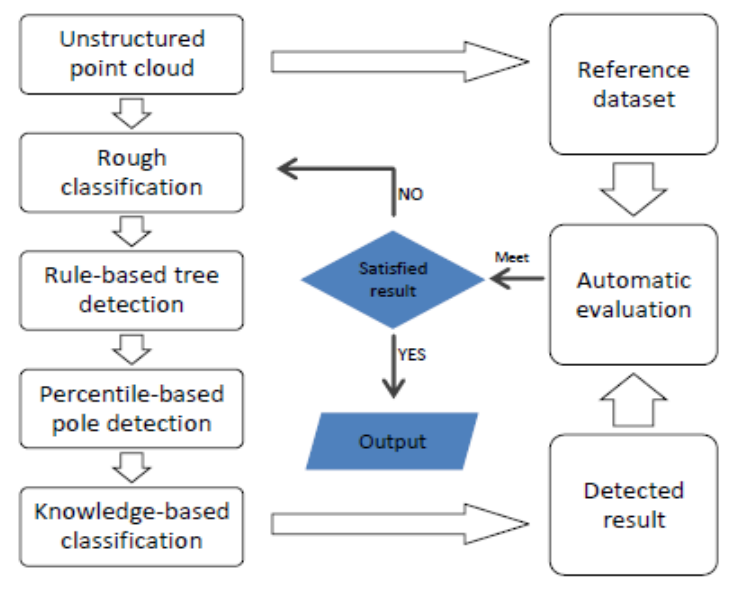

Figure 1: Workflow of the proposed methodology

\subsection{Rough classification}

The purpose of rough classification is to make the remaining points significantly less than the original data to reduce unnecessary computational consuming by removing points that are not the ones of interest. The improvement of rough classification is mainly on optimizing the segmentation based filtering parameters of two steps: smooth surface based segmentation and filtering criteria. The thresholds utilized in this step are: surface growing segmentation, connected component analysis and filtering criteria.

After surface growing segmentation, the laser points are grouped into different segments. Planar segments are usually the basic elements, which can compose more complex human made objects. Therefore the derived planar segments are well suited to serve as basic elements for recognition of ground surface and facades. For these reasons, the ground and building facades can be removed using the geometric attribute when it can fulfil constrains like: ground surface segments are probably the large planar surface below 3D trajectory of the laser scanner; building facades are assumed as large vertical planar surface which connected to the ground surface.

The planar segments are combined together as the ground surface after being detected. In the next step, only segments completely within 2D outline of ground surface and connect to it, are identified as on-ground segments. For such segments, a connected component analysis is utilized to group nearby points as component. Before extracting the pole-like objects after achieving component, it is necessary to further remove objects that not the ones of interest, i.e., cars, small building structures, bus shelters. Thus, filtering criteria is applied then. One section of each segment is automatically selected from component to check the diameter value (Figure 2). One segment can be labelled if the diameter is less than a given threshold value. At last, an assumption is proposed to label the entire component as a candidate pole-like object if part of it is labelled.

Because the ground and facade surface are easily detected in the laser data, parameters on the surface growing algorithm are not so critical and mild under-segmentation or over-segmentation can be tolerated.

The defects of this step are that: in the current algorithm, the segments not completely within 2D outline and off-ground points, which are actually candidate pole-like segments, have 
been removed incorrectly (such as object within white circle in Figure 3). Thus, the on-ground segments should be refined. In addition to this, several trees and lamp posts, some traffic signs were connected as one object because of the high connected component analysis threshold value setting. At last, the threshold value of the diameter constrain is too small to filter lots of trees and other potential pole-like objects.

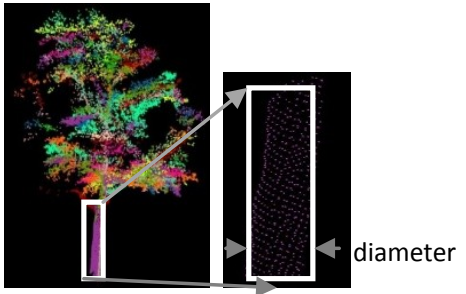

Figure 2: Each section is selected to check whether the segment can be labelled

Improvement: to improve the defects of the convex hull, through field observation and statistical analysis on the laser points, part of off-ground objects should be considered for better defining the on-ground segments. For this reason, not only the segments completely within the convex hull, but also the ones close to it and part of off-ground points should be classified as on-road segments. For optimizing the segmentation, statistical analysis on the parameter determination would be carried out to get a trade-off between under-segmentation and over-segmentation and also optimal diameter value to keep potential pole-like objects as much as possible.

\subsection{Rule-based tree detection}

Owing to some occluded and thick tree trunk, tree detection is difficult through current algorithm. A rule-based tree detection method is developed in this section to detect the tree which has no or relatively thick tree trunk in the point cloud, and then separate the tree from other on-road objects. In term of the human knowledge, the characteristics of natural object are different from the man-made one, a list of geometric and radiometric attributes are calculated for each component. Similar with the rule-based collapsed building detection in (Oude Elberink et al., 2011), different kinds of attributes are taken into consideration to build the rules.

Height: Based on statistical analysis, we find that the lamp post is often the highest object on road except for trees, which are supposed to be classified in the previous step.

Area: Area is a measure of area for the Minimum Bounding Rectangle (MBR) derives from the component after being projected onto 2D plane. The area of MBR is a sharp indicator can be used because the projections of traffic pole furniture usually shows long and narrow linear projection while tree has larger $2 \mathrm{D}$ projection owing to tree crown.

Ratio: As the reason mentioned above, the road traffic furniture shows long and narrow linear projection most times while trees have no regular shape but usually the length and width of MBR are relatively close. The ratio is an important index to extract tree.

Percentage of pulse count: In addition to the geometric attribute, radiometric information of laser point, especially the pulse count information is also an important factor for tree extraction. Vegetation has rough surface to causes multiple pulse count, while in most buildings, last and first pulse data are similar depending on the material of façade. During measurement of trees, certain percentage of a laser footprint would be reflected by the branches and leaves of trees, other parts will penetrate the foliage and finally be reflected by building at roadside. Therefore, the percentage of multiple pulse count points of tree should be higher than man-made objects.

Number of segments: After surface growing segmentation, since the unstructured distribution of points on vegetation, the number of segment in component of tree is clearly higher than man-made object.

After statistically analysing each type of objects in training areas, the attribute feature can be derived as input of developing decision tree for the rule-based detection. The automatic tree detection is based on a count system where a counter is defined to count the number of time meeting the above conditions for each component; at the end, sum up the count per component. Whether the component can be recognized as tree depends on the final count value: the component with the count value 3 or 4 can be recognized as a tree. As a result, a set of automatically detection rules from the decision tree are produced, and then these rules are used in the testing areas to detect trees.

\subsection{Percentile-based detection}

The purpose of this step is to determine optimal parameters of percentile-based algorithm by using statistical analysis. The principle of percentile-based pole detection algorithm is: (a). Divide the whole candidate component into several sections and select one as test part. (b). Slice section into multiple horizontal slices and check the deviation of centre position between neighbouring two slices (c). Check the length of diagonal and difference between two adjacent slices diagonals. A counter is defined to count once if these four values are within given threshold simultaneously. At last, if the count is greater than a given value, this selected section can be recognized as pole. As a result, such whole component is labelled as pole-like object. Five parameters are utilized in this step. Due to the harsh threshold values used in current algorithm, some poles were missing detected. However, through statistically analyzing the parameters in training areas, optimal threshold values were formulated to get more reliable result. As a result, most of the pole-like objects can be recognized using the improved percentile-based algorithm, and then be preserved as the input for further classification.

\subsection{Knowledge-based classification}

In (Pu et al., 2011), the method simply classifies the pole-like objects into four categories: poles, trees, road signs and others. More classes are classified, more beneficial it is to improve the actual transportation safety. Therefore, it is important to further identify the transport facilities such as traffic sign, traffic light or roadside furniture like lamp post. After removing the pole part from detected pole, several distinction attributes are analysed. Strong clues can be generated by the laser points with the feature description to determine the most likely pole type.

Size: The term size refers to the number of point per segments on the additional structures of pole. If the size of the remaining segment is less than 10 points, such segment can be classified as other pole, including flagpole, bare pole, etc. 
Shape: Shape can be used as an important element to differential types of pole, because man-made objects usually have regular and common shapes. In most cases, traffic sign is rectangle or circle, the top of traffic light and lamp post is long and narrow linear. The tree points are irregular and randomly distributed in 3D space.

Height: Based on statistical analysis, we find that the lamp post is often the highest object on road except for tree.

Reflectance intensity information: According to the international practice, for various applications in the nighttimes and low-light visibility, high reflection material is painted on the traffic signs. Since traffic sign contains number of points with significantly higher reflection value than others, the reflectance intensity information could be a significant indicator to extract the traffic sign.

Improvement: Due to the limitation of only using coordinate information in current algorithm, the further classification did not get an accurate result. However, thanks to the statistical analysis about reflectance strength information and several adopted geometric attributes, more classes and higher accuracy can be achieved by using this proposed algorithm.

\subsection{Evaluation}

After getting the detected poles, an automatic evaluation is carried out to show the performance of proposed algorithm. The evaluation is carried out by comparing detected pole-like objects with the reference dataset, which provides the ground truth of each testing area. Supposing that the detection accuracy is not satisfied, another optimization process would automatically start again till achieving optimal result.

\section{RESULT AND DISCUSSION}

We test the pole-like objects with the proposed algorithm, and get the detection accuracy on two datasets ${ }^{*}$, which contains 9 and 10 road parts separately, and are randomly selected from the original dataset. Since the dataset B does not contain the pulse count information (PCI), the performance of PCI is demonstrated by comparing result which considering and not considering it in dataset A. To remedy the lack of PCI in dataset $\mathrm{B}$, segment number in each component is applied in the rulebased tree detection. After formulating the optimal threshold values in percentile-based algorithm, five classes of pole are detected and distinguished based on detected poles. As a result, completeness, correctness, overall accuracy and confusion matrix are achieved. After formulating the performance, some issues concerning the algorithm are discussed.

\subsection{Determination of rule and optimal threshold}

Four road parts are selected as training areas in both two datasets. Since there is no PCI in Dataset B, after statistical analysis on such four parameters (Figure 4), optimal thresholds for rule-based detection are determined to build rule.

After the tree detection, three parameters which are used in percentile-based algorithm are statistical analyzed to get optimal threshold. The statistic analysis of such parameters is revealed in Figure 5.

\footnotetext{
A: Enschede dataset, B: Ireland dataset
}
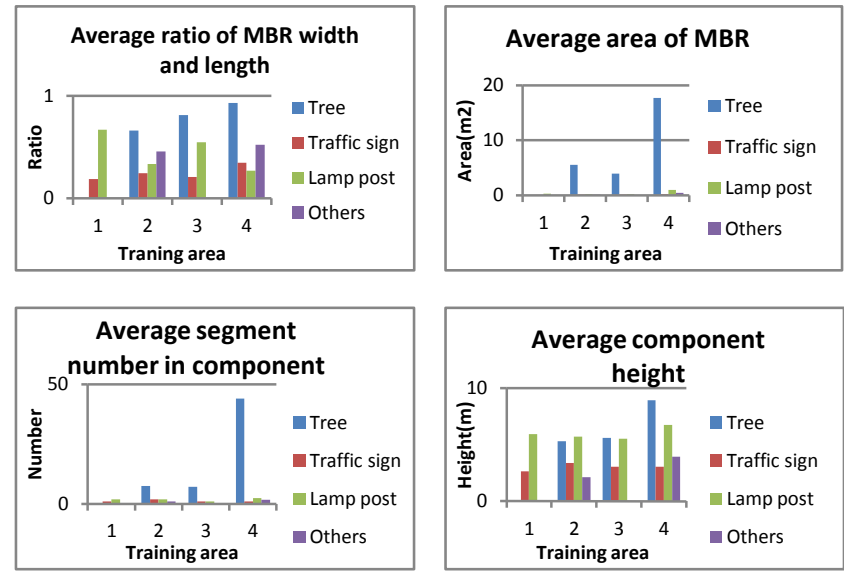

Figure 4: Statistic analysis on four parameters in rule-based algorithm in four training areas (dataset B)
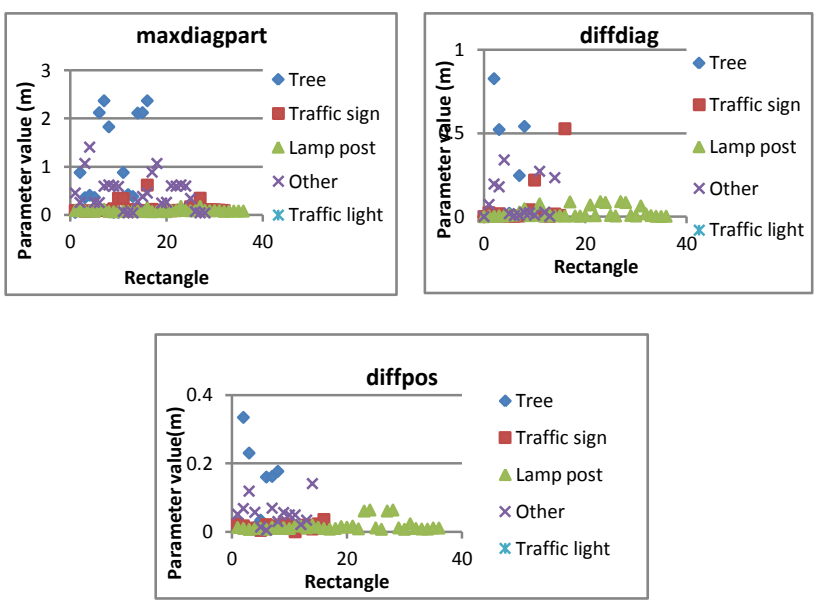

Figure 5: Statistic analysis on three parameters in percentilebased algorithm (dataset B)
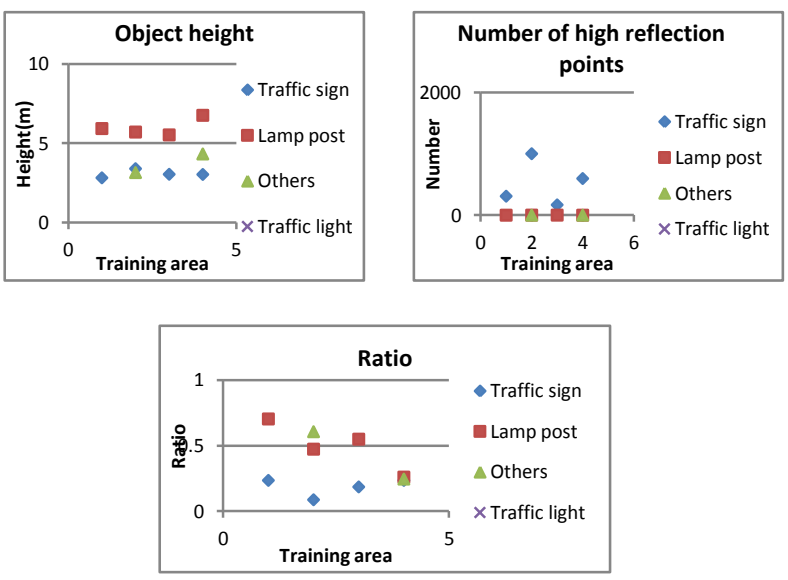

Figure 6: Statistic analysis on three parameters in knowledge-based classification (dataset B)

The next step is adopting a knowledge-based classification to distinct detected pole into specific types such as lamp post, traffic sign, traffic light or others. To differentiate kinds of polelike objects, the segments after removing the vertical pole part are analyzed to gain the hint for further classification. Based on such idea, several feature attributes are caculated to get optimal value as classification indicator (Figure 6). The final evluation 
result based on point wise of two dataset is tabulated in Table 1 and a visual result of detection pole is shown in Figure 7.

\begin{tabular}{|c|c|c|c|c|}
\hline \multicolumn{2}{|c|}{ Dataset } & Completeness & Correctness & Accuracy \\
\hline \multirow{2}{*}{ Dataset A } & Tree & 95.1 & 96.7 & 92.5 \\
\cline { 2 - 5 } & Pole & 80.5 & 88.2 & 72.4 \\
\hline \multirow{2}{*}{ Dataset B } & Tree & 92.3 & 88.7 & 82.3 \\
\cline { 2 - 5 } & Pole & 93.6 & 79.5 & 75.1 \\
\hline
\end{tabular}

Table 1: Evaluation result of on pole-like object detection based on automatic point wise

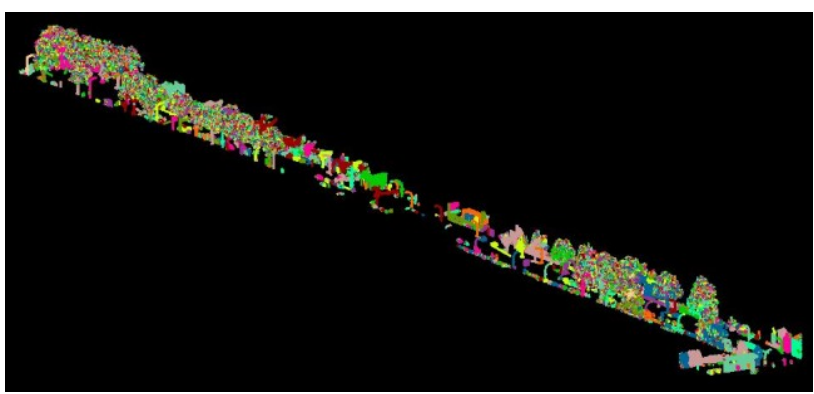

(a)

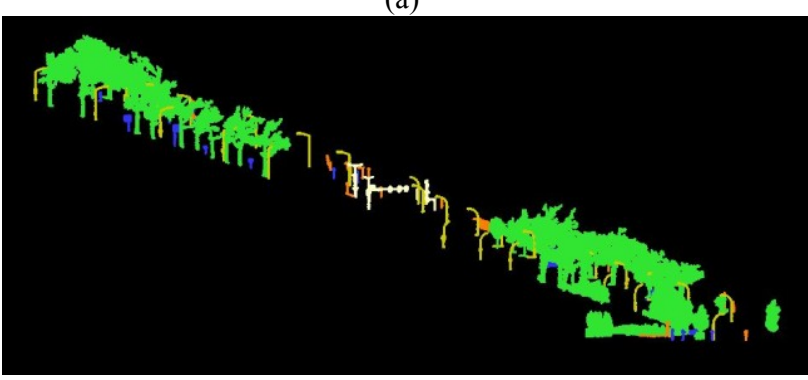

(b)

Figure 7: Result of the detection algorithm in dataset A, (a) input data (b) output classified result

\subsection{Evaluation and Discussions}

After the selection of parameter values, an automatic evaluation is carried out to show detection result automatically. At last, point-wise automatic evaluation and object-wise visual examination are adopted to show the performance.

\subsubsection{Pulse count information}

The importance of using PCI is demonstrated by comparing the dataset A with and without it. After optimizing each parameter in the proposed algorithm, the comparison is shown in Table 2. From this table, we can clear see the tree detection rate reduces obviously, and the false detection of traffic light increases. It means that some of the trees are falsely classified as traffic lights without PCI. As we know, the proposed tree detection algorithm relies on the PCI, projection area and ratio of tree crown. However, the adjacent several trees would be connected as one object without PCI, and ratio of width and length on the MBR becomes small and performs unlike pole. As the reason mentioned above, PCI is demonstrated to be an importance and efficient indicator to extract tree from other on-road objects.

\subsubsection{Segment number in component}

Since the unconstructed point distribution in tree, the point cloud is over-segmented into many segments. As a result, segment number in tree is clearly higher than other pole-like objects. To remedy the lack of PCI in dataset B, segment number information is adopted to replace PCI during tree detection. From Table 1 we can notice that since there is no PCI in dataset, the detection rate of tree reduces obviously when comparing with data contains it, however with segment number information instead of PCI, a basic acceptable detection rate $(87.2 \%)$ is obtained on the tree detection. As a result, segment number in component would be a significant indicator to extract tree from laser point cloud with the lack of PCI.

\begin{tabular}{|l|l|c|c|c|c|c|}
\hline \multicolumn{2}{|c|}{ Dataset A } & Tree & $\begin{array}{c}\text { Lamp } \\
\text { post }\end{array}$ & $\begin{array}{c}\text { Traffic } \\
\text { sign }\end{array}$ & $\begin{array}{c}\text { Traffic } \\
\text { light }\end{array}$ & $\begin{array}{c}\text { Othe } \\
\text { r }\end{array}$ \\
\hline \multirow{2}{*}{$\begin{array}{l}\text { Detection } \\
\text { rate }\end{array}$} & With PCI & 83.3 & 85.3 & 65.4 & 100 & 76.9 \\
\cline { 2 - 7 } & $\begin{array}{l}\text { Without } \\
\text { PCI }\end{array}$ & 66.7 & 85.3 & 65.4 & 100 & 76.9 \\
\hline \multirow{2}{*}{$\begin{array}{l}\text { False } \\
\text { detection }\end{array}$} & With PCI & 0 & 0 & 0 & 16.7 & 23.1 \\
\cline { 2 - 7 } & $\begin{array}{l}\text { Without } \\
\text { PCI }\end{array}$ & 2.7 & 0 & 0 & 33.3 & 23.1 \\
\hline
\end{tabular}

Table 2: Performance with \& without PCI based on object wise (dataset A)

\begin{tabular}{|c|c|c|c|c|c|c|c|}
\hline \multirow{2}{*}{\multicolumn{2}{|c|}{ Dataset B }} & \multicolumn{5}{|c|}{ Visual inspection } & \multirow{2}{*}{$\begin{array}{c}\text { False } \\
\text { positives } \\
(\%) \\
\end{array}$} \\
\hline & & Tree & $\begin{array}{c}\text { Lamp } \\
\text { post }\end{array}$ & Traffic sign & Others & $\begin{array}{c}\text { Total } \\
\text { detected }\end{array}$ & \\
\hline \multirow{7}{*}{ 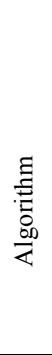 } & Tree & 41 & 0 & 0 & 0 & 41 & 0 \\
\hline & Lamp post & 0 & 10 & 0 & 1 & 11 & 9.1 \\
\hline & Traffic sign & 0 & 0 & 9 & 0 & 9 & 0 \\
\hline & Other & 3 & 0 & 1 & 11 & 15 & 26.7 \\
\hline & Missed & 3 & 1 & 2 & 6 & 12 & \\
\hline & Total visual & 47 & 11 & 12 & 18 & 88 & \\
\hline & $\begin{array}{l}\text { Detection rate } \\
(\%)\end{array}$ & 87.2 & 90.9 & 75 & 61.1 & & \\
\hline
\end{tabular}

Table 3: Confusion matrix of the detected result based on object wise (dataset B)

\subsection{Analysis and discussion}

Although the proposed algorithm has improved current algorithm in some extent, there are still several bottlenecks which reduce the accuracy of proposed algorithm. The problems of tree detection are that, the proposed tree detection algorithm relies on the pulse count information and projection area of tree crown. Since some trees only include a small amount of leaves in the tree crown, or several tree are connected as one object, at the same time the thick tree trunks exceed threshold values of the percentile-based pole detection, these kinds of trees are missing detected.

For the missing detection of other pole-like objects, because all of the poles of interest are considering the objects at least within $1 \mathrm{~m}$ above to the ground, some actual pole-like objects which hanging in the air are removed. Another reason of the missing pole detection is the point number between the selected slices. In the detection algorithm, one section was selected as considered section to determine whether this object is a pole or not. Because the fewer number of points in the selected section, it is harder to collect the rectangular in detection process, such as derive the minimum bounding rectangle for each subpart, or count the number of the rectangle, which should be at least 2 rectangles in the selected section. Meanwhile, since some selected sections are too short to collect enough rectangles, these pole-like objects have been missing detected. It is difficult to completely avoid such problem: if the section between two adjacent rectangular is selected as high as possible, there would not be sufficient number of rectangular can be utilized in pole detection. Another problem is that different types of pole-like objects are connected together as one component because they located very close to each other. For example, lamp post is 
connected together with nearby trees, which would lead to low detection rate and high false detection.

Last problem is about the further classification based on knowledge-based classification. Proposed algorithm gives some constrains for types of pole distinction. However, not all of the pole-like objects have regular shape on ground, and meanwhile not all of the same type of poles performs the same shape in different areas. It is a major challenge to design an algorithm that can work perfectly in all situations.

\section{CONCLUSION AND RECOMMENDATION}

The main objective of this research is to optimize detection algorithm of pole-like objects in mobile laser scanner data based on existing algorithm by (Pu et al., 2011). By proposing new rule-based tree detection algorithm, knowledge-based classification and optimizing each parameter threshold, an improved five-step pole detection algorithm is presented: rough classification, rule-based tree detection, percentile-based pole detection, knowledge-based classification and automatic evaluation. Although the proposed classification cannot success under all circumstances, it is still convincing that the proposed algorithm is feasible in the pole-like objects detection from MLS dataset.

The accuracy on tree detection of proposed algorithm is $92.5 \%$ and $82.3 \%$ in dataset $\mathrm{A}$ and $\mathrm{B}$, and $72.4 \%$ and $75.1 \%$ on other pole-like objects based on point wise. From these figures, we can notice that the proposed algorithm is validated to get basically accurate result.

However, because of the knowledge and time limitation, some aspects need further work and several recommendations are listed below based on current research: Because the lamp posts are often distributed regularly on roadside, the contextual information can be used to give a hint on the location of lamp post once it is connected with or occluded by tree. Due to much richer texture and colour information provided by imagery is beneficial to recognize the traffic sign based on knowledge recognition, the combination of laser points and imagery should be investigated in the future. At last, the proposed method can be tested by combining the pulse count information with segment number in component together to demonstrate whether it would be more accurate when using both of two attribute simultaneously.

\section{Reference}

Brenner, C. (2009). "Extraction of Features from Mobile Laser Scanning Data for Future Driver Assistance Systems", Advances in GIScience (pp. 25-42): In: Geoinformation and Cartography, Springer.

Darmawati, A. T. (2008). Utilization of Multiple Echo Information for Classification of Airborne Laser Scanning Data. Master's Thesis, International Institute for Geo-Information Science and Earth Observation (ITC), Enschede, the Netherlands.

El-Halawany, S. I., \& Lichti, D. D. (2011). Detection of Road Poles from Mobile Terrestrial Laser Scanner Point Cloud. On: Multi-Platform/Multi-Sensor Remote Sensing and Mapping (M2RSM), 2011 International Workshop.

Golovinskiy, A., Kim, V. G., \& Funkhouser, T. (2009, Sept. 29 2009-Oct. 2 2009). Shape-based recognition of 3D point clouds in urban environments. On: Computer Vision, 2009 IEEE 12th International Conference.

Lehtomäki, M., Jaakkola, A., Hyyppä, J., Kukko, A., \& Kaartinen, H. (2010). Detection of Vertical Pole-Like Objects in a Road Environment Using Vehicle-Based Laser Scanning Data. Remote Sensing, 2(3), 641-664.

Mc Elhinney, C., Kumar, P., Cahalane, C., \& McCarthy, T. (2010). Initial Results from European Road Safety Inspection (EURSI) Mobile Mapping Project. In: The International Archives of the Photogrammetry, Remote Sensing and Spatial Information Sciences, Newcastle upon Tyne, UK, Vol. XXXVIII, Part 5, 440-445.

Oude Elberink, S., Shoko, M., Fathi, S. A., \& Rutzinger, M. (2011). Detection of Collapsed Buildings by Classifying Segmented Airborne Laser Scanner Data. On: ISPRS Workshop Laser Scanning 2011, Calgary, Canada, 29-31 August 2011.

Pu, S., Rutzinger, M., Vosselman, G., \& Elberink, S. O. (2011). Recognizing Basic Structures from Mobile Laser Scanning Data for Road Inventory Studies. In: ISPRS Journal of Photogrammetry and Remote Sensing, 66(6), 28-39.

Rabbani, T., van den Heuvel, F. A., \& Vosselman, G. (2006). Segmentation of point clouds using smoothness constraint. In: The International Archives of the Photogrammetry, Remote Sensing and Spatial Information Sciences, Vol. XXXVI, Part 5, 248-253.

Rutzinger, M., Pratihast, A. K., Oude Elberink, S., \& Vosselman, G. (2010). Detection and Modelling of 3D Trees from Mobile Laser Scanning Data. In; The International Archives of the Photogrammetry, Remote Sensing and Spatial Information Sciences, Newcastle upon Tyne, UK, , Vol. XXXVIII, 520-525.

Vakautawale, M. (2010). Information Extraction from Mobile Laser Scanner for Road Inventory Application. Master's Thesis, International Institute for Geo-Information Science and Earth Observation (ITC), Enschede, the Netherlands.

Vosselman, G., Gorte, B. G. H., Sithole, G., \& Rabbani, T. (2004). Recognising Structure in Laser Scanner Point Clouds. In: The International Archives of the Photogrammetry, Remote Sensing and Spatial Information Sciences, Freiburg, Germany, Vol. XXXVI, Part 8/W2, 33-38.

Vosselman, G., \& Maas, H.-G. (2010). Airborne and terrestrial laser scanning. GB: Whittles Publishing.

Yokoyama, H., Date, H., Kanai, S., \& Takeda, H. (2011). Polelike Objects Recognition from Mobile Laser Scanning Data Using Smoothing and Principal Component Analysis. In: ISPRS Workshop Laser Scanning 2011, Calgary, Canada, 29-31 August 2011.

Yu, X., Hyyppä, J., Vastaranta, M., Holopainen, M., \& Viitala, R. (2011). Predicting individual tree attributes from airborne laser point clouds based on the random forests technique. In: ISPRS Journal of Photogrammetry and Remote Sensing, Vol. 66(1), 28-37. 